\title{
AVALIAÇÃO DOS PÉS E CLASSIFICAÇÃO DO RISCO PARA PÉ DIABÉTICO: CONTRIBUIÇÕES DA ENFERMAGEM*
}

Eduardo Godoi Audi ${ }^{1}$, Ricardo Castanho Moreira ${ }^{2}$, Ana Cândida Martins Grossi Moreira ${ }^{3}$, Eliana de Fátima Catussi Pinheiro $^{4}$, Maria de Fátima Mantovani ${ }^{5}$, Arlinda Gonçalves de Araújo ${ }^{6}$

RESUMO: O objetivo do estudo foi avaliar o grau de risco para pé diabético de pessoas com diabetes inscritas no Sistema HIPERDIA. Optou-se por uma abordagem quantitativa, do tipo epidemiológico-descritivo, cujos dados foram obtidos por meio de pesquisa de campo, de janeiro a abril de 2010. Foram selecionadas 50 pessoas com diagnóstico de diabetes, residentes da área de abrangência da Unidade Básica de Saúde do estudo. A maioria dos participantes foi de mulheres, na faixa etária entre 50 e 69 anos, com tempo de evolução da doença de 5 a 9 anos. O grau de risco 0 concentrou maior número de pessoas, com $56 \%$, porém houve aquelas que já apresentavam úlceras ou amputações em membros inferiores, sendo classificadas em risco 3. A estratificação do risco permite priorizar grupos de pessoas para melhor alocação dos recursos necessários para o cuidado em saúde.

PALAVRAS-CHAVE: Doença crônica; Pé diabético; Cuidados de enfermagem.

\section{ASSESSMENT OF FEET AND RISK CLASSIFICATION FOR DIABETIC FOOT: NURSING CONTRIBUTIONS}

\begin{abstract}
The objective was to study the degree of risk for diabetic foot among diabetics on the HIPERDIA system. An epidemiological-descriptive quantitative approach was chosen, with data obtained by field research between January and April 2010. Fifty people diagnosed with diabetes were selected for the study, all resident in the area attended by the Basic Health Unit of the study. The majority of participants were women, between the ages of 50 and 69 , who had suffered from diabetes for between 5 and 9 years. The degree of risk 0 included the largest number of people, with $56 \%$, although there were others who already had ulcers and lower member amputations, these people being classified as risk 3 . The risk stratification permits the prioritization of groups of people in order to better allocate the necessary health care resources.
\end{abstract}

KEYWORDS: Chronic Illness; Diabetic foot; Nursing care.

\section{EVALUACIÓN DE LOS PIES Y CLASIFICACIÓN DEL RIESGO PARA PIE DIABÉTICO: CONTRIBUCIONES DE LA ENFERMERÍA}

RESUMEN: El objetivo del estudio fue evaluar el grado de riesgo para pie diabético de personas con diabetes inscriptas en sistema HIPERDIA. Se eligió un abordaje cuantitativo, del tipo epidemiológico-descriptivo, cuyos datos fueron obtenidos por medio de investigación de campo, de enero a abril de 2010. Fueron seleccionadas 50 personas con diagnóstico de diabetes, residentes del área de abrangencia de la Unidad Básica de Salud del estudio. La mayoría de los participantes fue de mujeres, en la franja etaria entre 50 y 69 años, con tiempo de evolución de la enfermedad de 5 a 9 años. El grado de riesgo 0 concentró mayor número de personas, con $56 \%$, pero huvo aquellas que ya presentavan úlceras o amputaciones en miembros inferiores, siendo clasificadas en riesgo 3. La estratificación del riesgo permite priorizar grupos de personas para mejor distribución de los recursos necesarios al cuidado en salud.

PALABRAS CLAVE: Enfermedad crónica; Pie diabético; Cuidados de enfermería.

\footnotetext{
*Pesquisa financiada pela Fundação Araucária de Apoio ao Desenvolvimento Científico e Tecnológico do Paraná ${ }^{1}$ Acadêmico de Enfermagem na Universidade Estadual do Norte do Paraná - UENP. Bolsista de iniciação científica pela Fundação Araucária. ${ }^{2}$ Enfermeiro. Professor da UENP. Doutorando no Programa de Pós-Graduação em Enfermagem da Universidade Federal do Paraná-PPGENF UFPR. Membro do Grupo de Estudos Multiprofissional em Saúde do Adulto - GEMSA.

${ }^{3}$ Enfermeira. Professora da UENP. Mestranda no Programa de Pós-Graduação em Enfermagem da Universidade Estadual de Maringá.

${ }^{4}$ Enfermeira. Professora da UENP. Especialista em Saúde Pública. Mestranda em Educação na Universidade do Oeste Paulista.

${ }^{5}$ Enfermeira. Doutora em Enfermagem. Professora do Departamento e PPGENF UFPR. Membro do GEMSA.

${ }^{6}$ Enfermeira. Coordenadora da Equipe de Saúde da Família da Secretaria Municipal de Saúde de Bandeirantes, Paraná.
}

Autor correspondente:

Ricardo Castanho Moreira

Universidade Estadual do Norte do Paraná

Av. Prefeito Moacyr Castanho, 1483 - 86360-000 - Bandeirantes-PR-Brasil

Recebido: $24 / 11 / 10$

E-mail: ricardocastanho@uenp.edu.br

Aprovado: 20/04/11

Cogitare Enferm. 2011 Abr/Jun; 16(2):240-6 


\section{INTRODUÇÃO}

O Consenso Internacional sobre Pé Diabético o define como estado de infecção, ulceração e/ou destruição de tecidos moles associados a alterações neurológicas e vários graus de doença arterial periférica nos membros inferiores ${ }^{(1)}$. Estima-se em $25 \%$ a incidência de ulceração ao longo da vida entre portadores de diabetes, $85 \%$ das úlceras precedem a amputação(2), a qual tem se configurado como opção terapêutica, atingindo $52,2 \%$ dos casos. Tal casuística tem base na revisão bibliográfica realizada em periódicos publicados na base de dados do LILACS e SciELO, no período de 1992 a $2005^{(3)}$.

Esta complicação vem preocupando os profissionais do setor saúde, e a sua prevenção se tornou um desafio, haja vista que $57 \%$ das cirurgias de amputação realizadas entre 2009 e o primeiro semestre de 2010, no Hospital Universitário de Londrina, envolviam pacientes diabéticos ${ }^{(4)}$. Dados semelhantes foram encontrados no Hospital de Clínicas da Universidade Federal do Triângulo Mineiro, em pesquisa realizada em prontuários de pacientes submetidos a procedimento cirúrgico de amputação, no período de 2000 a 2005 , quando se constatou que $53,93 \%$ das cirurgias estavam associadas ao diabetes ${ }^{(5)}$.

Há também que destacar que a sobrevida do paciente diminui muito após a amputação, sendo de $65 \%$ em três anos e $41 \%$ após cinco anos ${ }^{(6)}$.

O Grupo de Trabalho Internacional sobre Pé Diabético reforça a importância dos profissionais de saúde neste processo, visto que o exame dos pés é quase sempre negligenciado, apesar das claras diretrizes e recomendações. Exames incompletos nos pés são relatados em até $50 \%$ dos pacientes que se submeteram a amputações ${ }^{(1)}$.

A Sociedade Brasileira de Diabetes destaca, como medida importante para prevenção secundária em diabetes, a prevenção de ulcerações nos pés e de amputações de membros inferiores por meio de cuidados específicos que podem reduzir tanto a frequência e a duração de hospitalizações como a incidência de amputações em $50 \%{ }^{(2)}$.

O Grupo de Trabalho Internacional sobre Pé Diabético orienta estes cuidados em cinco estratégias: inspeção e exame regular dos pés; identificação do pé em risco; educação dos familiares, pacientes e profissionais; uso de calçado adequado; e tratamento da patologia não ulcerativa ${ }^{(1)}$.

Considerando que a identificação do pé em risco, mediante inspeção e exame regular dos pés, é uma das estratégias iniciais para prevenção, nesta pesquisa o risco para pé diabético configurou-se como objeto de estudo e para descrevê-lo parte-se da seguinte questão norteadora: qual é a classificação do risco para manifestação do pé diabético de pessoas com diabetes inscritas no HIPERDIA, em uma Unidade Básica de Saúde?

Frente ao exposto, o objetivo desta pesquisa é avaliar o grau de risco para pé diabético de pessoas com diabetes inscritas no Sistema HIPERDIA, em uma Unidade Básica de Saúde.

\section{METODOLOGIA}

Trata-se de uma pesquisa com abordagem quantitativa, descritiva, cujos dados foram obtidos por meio de pesquisa de campo, no período de janeiro a abril de 2010.

A pesquisa foi realizada no Município de Bandeirantes, localizado na Região Norte do Estado do Paraná; a unidade escolhida foi uma Unidade Básica de Saúde (UBS) que atende a uma população de aproximadamente 4736 habitantes cadastrados, sendo destes, 175 pessoas portadoras de diabetes, conforme dados obtidos em 2008 pelo Sistema de Informação de Atenção Básica (SIAB).

Os participantes da pesquisa foram 50 pessoas com diagnóstico de diabetes, residentes na área de abrangência da Estratégia Saúde da Família (ESF) da Vila Lordani e selecionados por facilidade de acesso. A amostra representou $28,57 \%$ da população; os critérios de inclusão foram: ter recebido o diagnóstico de diabetes tipo 1 ou 2 e estar inscrito no plano de reorganização da atenção básica a hipertensão arterial e ao diabetes; ter 31 anos ou mais. Foram critérios de exclusão: pessoas com diabetes gestacional; pessoas ausentes da residência no dia da visita; e a recusa em participar da pesquisa. Não houve exclusão de sujeitos.

Para coleta de dados utilizou-se a consulta de enfermagem, e as pessoas foram avaliadas em seus domicílios. O instrumento elaborado solicitava as seguintes informações sociodemográficas: gênero e faixa etária; quanto aos dados clínicos: data do diagnóstico, tratamento medicamentoso, glicemia capilar, conhecimento sobre pé diabético, profissional que realizou a orientação, tipo de calçado e meia, amputação ou úlcera prévia, calosidade, deformidades ósseas, onicomicose, micose interdigital, pulsos periféricos, sensibilidade plantar ao monofilamento de 10 gramas e grau de risco para pé diabético.

Ao monofilamento de Semmes-Weinstein de 10 
gramas para o exame de sensibilidade do pé foram testados os seguintes locais: I, III, e V artelho; I, III e $\mathrm{V}$ cabeças metatarsianas, médio pé e região calcânea. $\mathrm{O}$ participante foi familiarizado previamente com o teste mediante explicações dos pesquisadores e este foi aplicado na mão ou cotovelo para conhecimento do que seria testado. A aplicação do monofilamento foi realizada com a pessoa sentada e com as pernas cruzadas, ou em decúbito dorsal e com os olhos fechados.

A aplicação foi feita com o monofilamento perpendicular à pele, até curvá-lo, e assim mantido por dois segundos, para então verificar se houve, ou não, sensibilidade ao mesmo. A percepção sensorial protetora foi considerada prejudicada quando não houve percepção do toque em dois ou mais pontos do pé(1).

Este exame fornece informação para a classificação do risco para pé diabético, em uma graduação de zero a três. O grau 0 significa neuropatia ausente, 1- neuropatia presente, 2- neuropatia presente, sinais de doença vascular periférica e/ou deformidades nos pés, e 3- presença de amputação ou úlceras prévias ${ }^{(1)}$.

Esta pesquisa foi aprovada pelo Comitê de Ética em Pesquisa com Seres Humanos do Curso de Enfermagem da Universidade Estadual do Norte do Paraná, campus Luiz Meneghel, Bandeirantes - Paraná, com o parecer n. 074-09, e teve a autorização do gestor municipal de saúde para sua realização na unidade de saúde referida. Somente participaram do trabalho aquelas pessoas que expressaram voluntariamente o consentimento, após leitura explicativa do termo de consentimento livre e esclarecido, conforme prerrogativa da Resolução no 196/96 do Conselho Nacional de Saúde.

Os dados foram analisados por medidas estatísticas descritivas, incluindo números absolutos, média, mediana, valor mínimo, valor máximo e proporção.

\section{RESULTADOS}

A amostra deste estudo foi constituída por 50 participantes, sendo a maioria do sexo feminino, perfazendo um total de $62 \%$. A faixa etária e o tempo de evolução com o diagnóstico são apresentados na tabela 1.

A figura 1 mostra os resultados obtidos com o teste de glicemia capilar casual dos participantes da pesquisa. Este teste tem como meta, para o controle do diabetes, resultado abaixo de $141 \mathrm{mg} / \mathrm{dL}^{(7)}$. A média dos resultados obtidos na amostra foi de 245 $\mathrm{mg} / \mathrm{dL}$, oscilando entre a mínima de $57 \mathrm{mg} / \mathrm{dL}$ e a máxima de $600 \mathrm{mg} / \mathrm{dL}$. A mediana foi de $226,5 \mathrm{mg} /$ dL; $11(22 \%)$ sujeitos estavam com valores abaixo de $140 \mathrm{mg} / \mathrm{dL}$. Entretanto, a maioria dos valores do teste estava acima do valor recomendado para o bom controle glicêmico.

Com relação ao tratamento medicamentoso, 9 (18\%) pessoas afirmaram não tomar medicamento algum para controle do diabetes, e destas, cinco (10\%) apresentaram glicemia acima de $140 \mathrm{mg} / \mathrm{dL}$.

Tabela 1 - Caracterização dos sujeitos da pesquisa de acordo com a faixa etária e tempo de evolução com o diagnóstico de diabetes. Bandeirantes, 2010

\begin{tabular}{lcc}
\hline \multicolumn{1}{c}{ Característica } & N & \% \\
\hline Faixa etária & & \\
\hline $31-50$ anos & 07 & 14 \\
$50-70$ anos & 24 & 48 \\
$70-90$ anos & 19 & 38 \\
\hline Total & 50 & 100 \\
\hline Tempo de evolução com o diagnóstico & & \\
\hline $0-1$ ano & 04 & 08 \\
$1-5$ anos & 16 & 32 \\
$5-10$ anos & 17 & 34 \\
10 anos ou mais & 13 & 26 \\
\hline Total & 50 & 100 \\
\hline
\end{tabular}

O conhecimento dos participantes sobre o que é pé diabético e se já tinham recebido orientações foi investigado e pode-se verificar que $78 \%$ relataram desconhecer esta complicação e, logo, as necessidades ao autocuidado. Às pessoas que afirmaram ter participado do processo de ensino-aprendizagem lhes foi solicitado que indicassem o profissional envolvido na construção deste saber, $54 \%$ apontaram ser o enfermeiro, $28 \%$ o médico, $9 \%$ enfermeiro e médico e $9 \%$ membros de instituições reliosas.

A avaliação dos calçados e meias que eles relataram usar com maior frequência pode ser visualizada na figura 2. Observa-se que mais da metade das pessoas com diabetes não utilizava calçados e meias adequadas, comportamento que expõe os pés a traumas extrínsecos.

Com relação à avaliação dos membros inferiores, notaram-se as características apresentadas na figura 3. Destaca-se que $16 \%$ dos pacientes apresentavam úlceras ou possuíam amputações, conferindo proporção aproximada de uma pessoa com úlcera ou amputação a cada seis pessoas avaliadas.

A avaliação da qualidade dos pulsos periféricos mostrou que $22 \%$ dos participantes da pesquisa tinham o pulso patelar posterior e podálico diminuídos, e $24 \%$ apresentavam o pulso tibial posterior diminuído.

Com relação à percepção sensorial na região plantar avaliada pela sensibilidade ao monofilamen- 
Figura 1 - Distribuição dos participantes da pesquisa de acordo com o resultado do teste de glicemia capilar. Bandeirantes, 2010

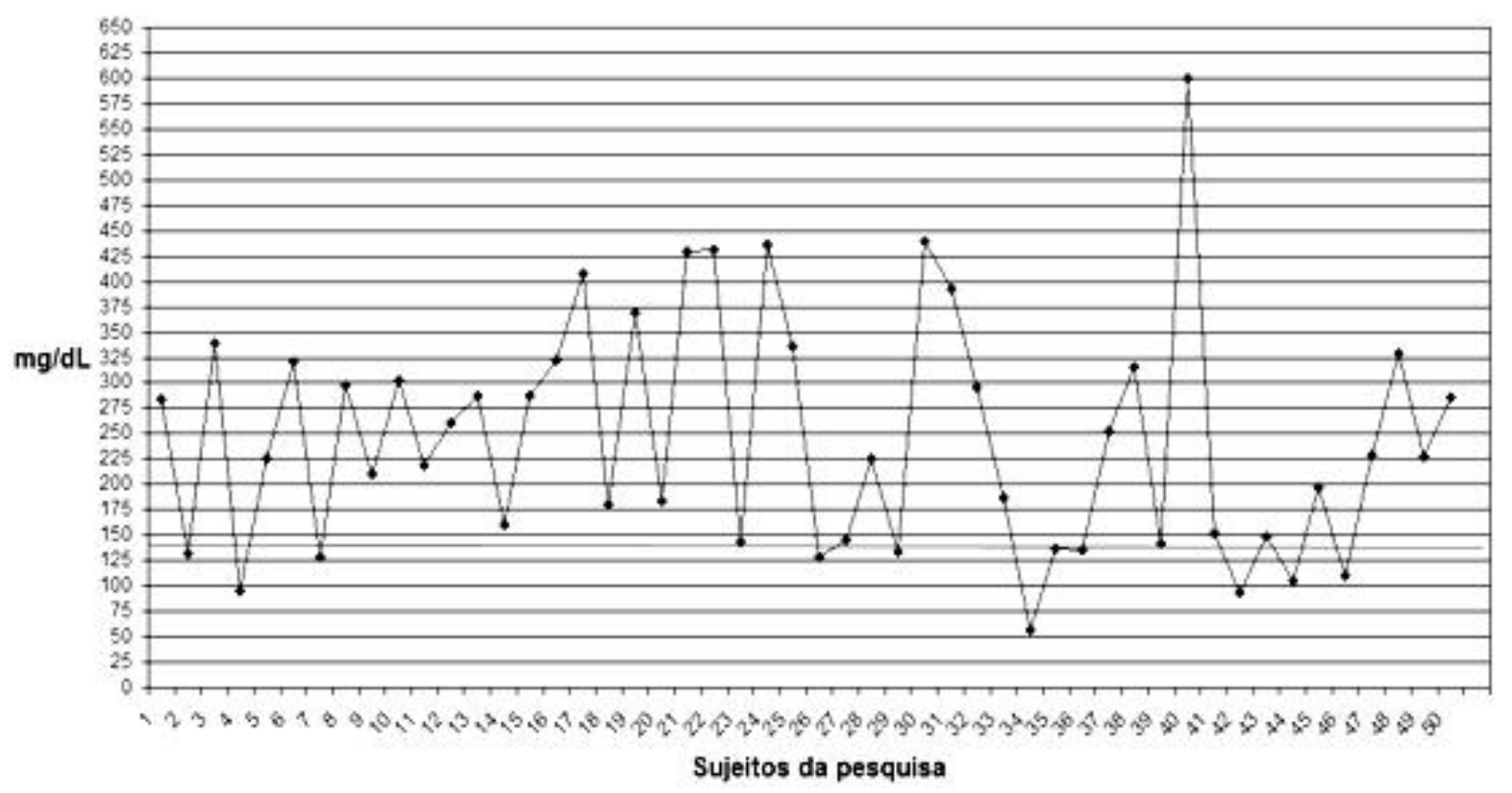

to de Semmes-Weisten de 10 gramas, constatou-se que houve maior porcentagem de insensibilidade na região calcânea, com $40 \%$. Outros pontos que apresentaram maior evidência de insensibilidade foram cabeça do primeiro metatarso, com $33 \%$, e o hálux, com $22 \%$.

Esta avaliação foi necessária para estratificação do risco para pé diabético, a qual é ilustrada na tabela 2. DISCUSSÃO
Tabela 2 - Distribuição dos participantes da pesquisa de acordo com a classificação do grau de risco para pé diabético. Bandeirantes, 2010

\begin{tabular}{lcc}
\hline Classificação do risco & $\mathbf{N}^{\mathbf{0}}$ & $\mathbf{\%}$ \\
\hline Risco 0 & 28 & 56 \\
Risco 1 & 12 & 24 \\
Risco 2 & 02 & 04 \\
Risco 3 & 08 & 16 \\
\hline Total & 50 & 100 \\
\hline
\end{tabular}

Figura 2 - Distribuição dos participantes da pesquisa de acordo com a avaliação dos calçados e meias. Bandeirantes, 2010
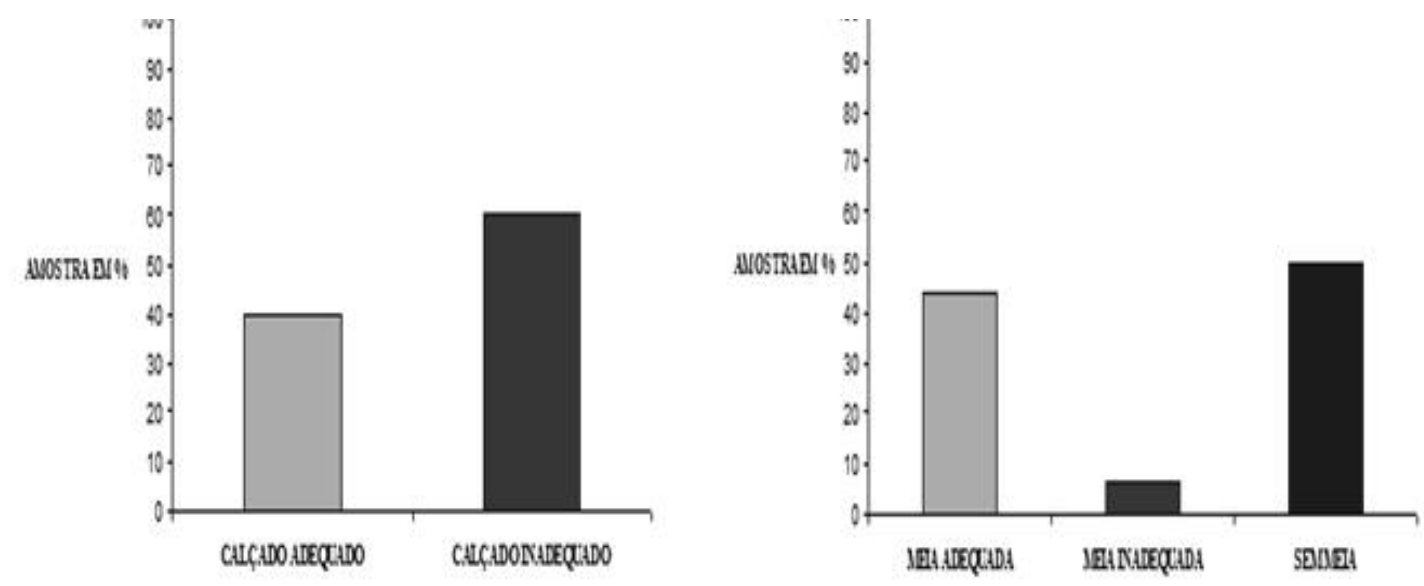
Figura 3 - Distribuições da proporção dos fatores de risco evidenciados nos participantes da pesquisa, durante a inspeção e palpação dos pés. Bandeirantes, 2010

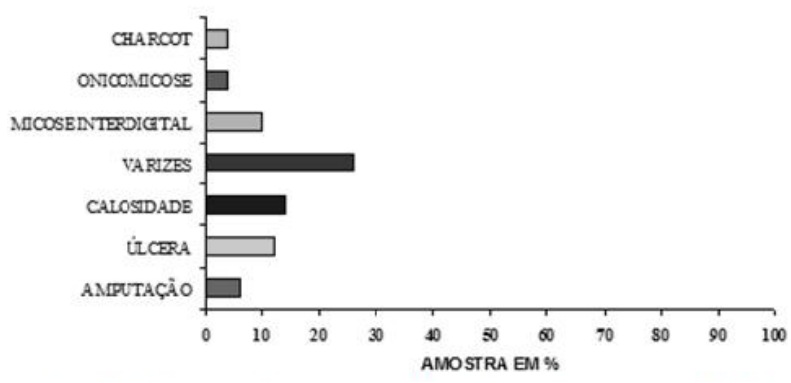

A faixa etária e o tempo do diagnóstico são variáveis que estão relacionadas, respectivamente, ao aumento da prevalência do diabetes e da ocorrência de complicações, sendo importantes para caracterização da população. A faixa etária predominante foi de 51 a 70 anos, e o maior percentual do tempo de evolução com o diagnóstico esteve entre 6 a 10 anos. Evidências de complicações micro e macrovasculares podem aparecer após o paciente evoluir com hiperglicemia não detectável entre 4 e 7 anos, e ainda, o fator idade acentua a gravidade de complicações vasculares, podendo acarretar as úlceras ${ }^{(7)}$.

$\mathrm{O}$ descontrole glicêmico representa outro fator de risco associado ao pé diabético, pois a taxa de glicose acima de $200 \mathrm{mg} / \mathrm{dL}$ representa um fator de risco independente para amputação ${ }^{(8)}$. Isso porque a hiperglicemia persistente leva ao acúmulo de produtos da via dos poliois (como sorbitol e frutose) nos nervos, causando lesões através de um mecanismo ainda não muito bem conhecido. A diminuição da incorporação da bomba $\mathrm{Na} / \mathrm{K} / \mathrm{ATPa} e$ resulta em retenção de sódio, edema da bainha de mielina, disjunção axoglial e degeneração nervosa.

Uma pesquisa documental, realizada em prontuários de pacientes com pé diabético admitidos em uma clínica vascular de um hospital de grande porte de Recife (PE) mostrou que eles apresentavam média de glicemia na admissão de $212 \mathrm{mg} / \mathrm{dL}$. Para aqueles que se submeteram à amputação, foi constatada associação com taxa de glicemia elevada, ratificando a informação anterior $^{(9)}$.

Com relação ao tratamento medicamentoso, 9 pessoas referiram não fazer uso de antidiabéticos, ainda que apresentando resultado da glicemia capilar acima da meta para o controle da enfermidade. Para estas, orientou-se a adoção de hábitos alimentares saudáveis, atividades físicas e o monitoramento de enfermidade pelo teste de glicemia capilar, realizado na UBS de seu bairro. Na UBS, o indivíduo tem a garantia da continuidade dos cuidados e o acesso ao atendimento pelos profissionais enfermeiro e médico da família, bem como pelo médico endocrinologista, quando necessário ${ }^{(7)}$.

O enfermeiro foi o profissional mais citado como agente do processo de educação em saúde, fato que evidencia a importância deste profissional na prevenção do pé diabético. Autores consideram que o enfermeiro tem contato mais próximo e regular com o paciente, empenhando-se no processo de aprendizagem $^{(10)}$.

Outros autores salientam a importância da inclusão dos integrantes da família no processo educativo, pois estes não só ajudam a identificar fatores de risco para complicações, mas também estabelecem uma rede apoio que melhora o efeito da educação no controle metabólico ${ }^{(11)}$.

A equipe da assistência primária deve trabalhar na conscientização das necessidades e riscos a que estão sujeitas as pessoas com diabetes em desenvolver o pé diabético. Desta maneira, a educação e a promoção de medidas simples, como sapatos apropriados e higiene dos pés, contribuem para a prevenção de úlceras, minimizando a influência dos riscos e o número de amputações ${ }^{(12)}$. Porém, nem todos os indivíduos seguem esta recomendação, como demonstrado na figura 2.

Notou-se que a maioria dos participantes não usava calçado adequado. O Grupo de Trabalho Internacional de Sobre Pé Diabético ${ }^{(1)}$ reforça esta preocupação ao alertar que a adesão ao uso de calçados adequados é um problema significativo e frequente entre as pessoas com diabetes. Reforçando esta constatação, resultados como uma pesquisa realizada em uma UBS de Minas Gerais demonstraram que 92\% dos diabéticos lá atendidos não utilizavam calçados adequados $^{(13)}$. Porém, o estudo não apontou resultados com relação ao uso de meias.

Vale ressaltar que o uso de meias adequadas previne o aparecimento de micoses interdigitais e onicomicose, condições que agravam ainda mais o pé diabético. Ressalta-se também que quando há disponibilidade de calçados protetores, a prevenção da recorrência de úlceras é obtida entre 60 e $85 \%$ dos pacientes $^{(1)}$.

Uma pesquisa nacional, desenvolvida no Municí- 
pio de Porto Alegre (RS), com delineamento de coorte prospectiva, randomizada, tendo como intervenção a proposta da educação terapêutica e o fornecimento de calçados ortopédicos, com seguimento de dois anos, mostrou que o grupo que recebeu a intervenção apresentou menores taxas de ocorrência e recorrência de úlceras plantares, reforçando a importância do uso de calçados adequados ${ }^{(14)}$.

Os fatores de risco do pé diabético mais incidentes foram: varizes, calosidades, úlceras, micose interdigital e amputação. Em um estudo retrospectivo realizado no ambulatório de diabetes da Santa Casa de Belo Horizonte $(\mathrm{MG})^{(15)}$, a proporção de indivíduos com presença de calosidades foi três vezes maior que a ocorrência neste estudo. Diferença ainda maior é encontrada quando se observa a incidência de micose interdigital de $40 \%$ contra $4 \%$, na população deste estudo. Comparando com outra pesquisa, desta vez realizada no ambulatório do Hospital de Base de São José do Rio Preto (SP) ${ }^{(16)}$, os resultados foram mais semelhantes no tocante ao histórico de amputação, sendo na ordem de $5 \%$. No item presença de úlcera, a observação foi discrepante, pois o percentual encontrado nesta pesquisa foi duas vezes maior quando comparado aos dados da do Hospital de Base.

Com relação à circulação periférica, a ausência da detecção dos pulsos distais de membros inferiores representou outro fator de risco para pé diabético, visto que, em estudo anterior, a redução ou ausência de fluxo sanguíneo nas artérias periféricas estava intimamente relacionada ao desfecho de amputações ${ }^{(17)}$.

No que diz respeito à avaliação da sensibilidade protetora, a região calcânea sobressaiu-se como aquela, dentre as demais regiões plantares avaliadas, que obteve maior proporção de insensibilidade ao monofilamento de 10 gramas, atingido $40 \%$. Isto se deve a três principais características: ser uma área que apresenta maior quantidade de queratina e gordura; por ela ser, muitas vezes, uma das áreas mais sobrecarregadas durante a locomoção; e receber inervação sensitiva do nervo sural, o primeiro a ser atingido pela ocorrência da neuropatia ${ }^{(18)}$. Este resultado é similar à pesquisa realizada por pesquisadores do Departamento de Fisioterapia, Fonoaudiologia e Terapia Ocupacional, da Faculdade de Medicina da Universidade de São Paulo (SP), que comparou os déficits sensório-motores de pés de pacientes neuropatas, diagnosticados clinicamente como sujeitos saudáveis ${ }^{(18)}$.

Outros pontos que apresentaram maior evidência de insensibilidade foram áreas que também sofrem sobrecargas durante a locomoção, gerando áreas de hiperpressão e predispondo, desta forma, o aparecimento de úlceras plantares.

Verificou-se, na tabela 2, que a maioria (56\%) dos participantes não possuía neuropatia periférica, apresentando, portanto, a sensibilidade preservada, sendo classificadas como risco 0 . Entretanto, o estudo realizado em UBS da Cidade de Ribeirão Preto (SP) mostrou um percentual de $85,1 \%$ de pessoas com sensibilidade preservada ${ }^{(19)}$. Por outro lado, pesquisa realizada com pessoas com diabetes inscritas em uma UBS de Maceió (AL) evidenciou proporções menores, sendo que $49,5 \%$ das pessoas se apresentavam sem alterações de sensibilidade ${ }^{(17)}$.

Dos participantes que foram classificados no risco 3, a maioria relatou não ter recebido orientações sobre cuidado com os pés, o que repercutiu no uso de calçado inadequado por este grupo. Isto mostra que as pessoas com diabetes têm necessidade de ações educativas visando a prevenção do pé diabético e, muitas vezes, procuram os serviços de saúde apenas quando apresentam lesões em membros inferiores em estágio avançado ${ }^{(20)}$, o que contribui para necessidade de um tratamento mutilador.

\section{CONCLUSÃO}

O presente estudo permitiu estratificar os participantes de acordo com o grau de risco para pé diabético e proporcionar-lhes o cuidado que necessitavam, em parceria com a UBS.

A contribuição desta pesquisa legitima-se pela importância que a classificação em categorias de risco assume para o planejamento na alocação de recursos materiais e humanos, bem como no direcionamento das ações de cuidado junto a pessoas com diabetes, de acordo com suas necessidades, o que contribui para uma prática de gestão dos recursos públicos de forma equânime.

Os resultados apontam para a necessidade de maior investimento em estratégias de educação em saúde, atendimentos preventivos e programados por profissionais da saúde, e fornecimento de calçados terapêuticos e protetores.

A carência de conhecimento sobre a importância do uso de calçados e meias adequadas na prevenção do pé diabético pode contribuir para a ocorrência de ulcerações, todavia, a relação entre estas variáveis poderá ser tema de estudos futuros.

\section{AGRADECIMENTOS}


À Fundação Araucária, pelo apoio e confiança conferida nesta pesquisa e aos profissionais da Secretaria Municipal de Saúde de Bandeirantes.

\section{REFERÊNCIAS}

1. Grupo de Trabalho Internacional Sobre Pé Diabético. Consenso Internacional Sobre Pé Diabético. Brasília: Secretaria de Estado da Saúde do Distrito Federal; 2001.

2. Sociedade Brasileira de Diabetes. Diretrizes da Sociedade Brasileira de Diabetes 2009. $3^{\text {a }}$ ed. Itapevi: A. Araújo Silva Farmacêutica; 2009.

3. Hirota CMO, Haddad MCL, Guariente MHDM. Pé diabético: o papel do enfermeiro no contexto das inovações terapêuticas. Ciênc Cuid Saúde. 2008;7(1):114-20.

4. Borges F. Pé em risco: cresce número de amputações entre vítimas do diabetes. Folha de Londrina. 26 jul. 2010; Folha saúde:9.

5. Tavares DMS, Dias FA, Araújo LR, Pereira GA. Perfil de clientes submetidos a amputação relacionada ao diabetes mellitus. Rev Bras Enferm. 2009;62(6):825-30.

6. Jorge SA, Dantas SAPE. Abordagem multiprofissional do tratamento de feridas. São Paulo: Atheneu; 2003.

7. Sociedade Brasileira de Diabetes. Consenso Brasileiro sobre Diabetes 2002: diagnóstico e classificação do diabetes melito e tratamento do diabetes melito tipo 2 . São Paulo: Diagraphic; 2003.

8. Shojaiefard A, Khorgami Z, Larajini B. Independent risk factors for amputation in diabetic foot. Int $\mathrm{J}$ Diabetes Dev Ctries. 2008;28(2):32-7.

9. Santos ICRV. Atenção à saúde dos portadores de pé diabético: prevalência de amputações e assistência preventiva na rede básica de saúde [tese]. Rio de Janeiro (RJ): Fundação Oswaldo Cruz; 2008.

10. Balduino AFA, Mantovani MF, Lacerda MR. O processo de cuidar de enfermagem ao portador de doença crônica cardíaca. Esc Anna Nery. 2009;13(2):342-51.

11. Guzmám-Pérez MI, Cruz-Cavich AJ, Parra-Jiménez J, Manzano-Osorio M. Control glicémico, conociemientos y autocuidado de pacientes diabéticos tipo 2 que asisten a sesiones educativas. Rev Enferm IMSS. 2005;13(1):9-13.
12. Ochoa-Vigo K, Pace AE. Pé diabético: estratégias para prevenção. Acta Paul Enferm. 2005;18(1):100-9.

13. Carvalho BDP, Carvalho CDP, Martins DA. Aplicação dos cuidados com os pés entre portadores de diabetes mellitus. Cogitare Enferm. 2010;15(1):106-9.

14. Cisneros LL. Avaliação de um programa para prevenção de úlceras neuropáticas em portadores de diabetes. Rev Bras Fisioter. 2010;14(1):31-7.

15. Calsolari MR, Castro RF, Maia RM, Maia FCP, Castro AV, Reis R, et al. Análise retrospectiva dos pés de pacientes diabéticos do ambulatório de diabetes da Santa Casa de Belo Horizonte, MG. Arq Bras Endocrinol Metab. 2002;46(2):173-6.

16. Laurindo MC, Recco DC, Roberti DB, Rodrigues CDS. Conhecimento das pessoas diabéticas acerca dos cuidados com os pés. Arq Ciênc Saúde. 2006;12(2):80-4.

17. Assumpção EC, Pitta GB, Macedo CL, Mendonça GB, Albuquerque LCA, Lyra LCB. Comparação dos fatores de risco para amputações maiores e menores em pacientes diabéticos de um Programa de Saúde da Família. J Vasc Bras. 2009;8(2):133-8.

18. Sacco ICN, Sartor CD, Gomes AA, João SMA, Cronfli R. Avaliação funcional de diabéticos neuropatas. Rev Bras Fisioter. 2007;11(1):27-33.

19. Ochoa-Vigo K, Torquato MTCG, Silvério IAS, Queiroz FA, Torre-Ugarte-Guanilo MC, Pace AE. Caracterização de pessoas com diabetes em unidades de atenção primária e secundária em relação a fatores desencadeantes do pé diabético. Acta Paul Enferm. 2006;19(3):296-303.

20. Bona SF, Barbosa MAR, Ferraz CLH, Guarita LKS, Nina RVAH, Barbosa NMRF, et al. Prevalência do pé diabético nos pacientes atendidos na emergência de um hospital público terciário de Fortaleza. Rev Soc Bras Clin Med. 2010:8(1):1-5. 